\title{
INFLUÊNCIA DE UM PROGRAMA DE ATIVIDADES FÍSICAS DE LONGA DURAÇÃO SOBRE SINTOMAS DEPRESSIVOS EM IDOSAS
}

\author{
Vanessa Helena Santana Dalla Déa \\ Universidade Federal de Goiás, Goiânia, Goiás, Brasil. \\ Edilson Duarte \\ Universidade Estadual de Campinas, Campinas, São Paulo, Brasil. \\ José Rubens Rebelatto \\ Universidade Federal de São Carlos, São Carlos, São Paulo, Brasil.
}

\section{Alessandra Castro de Paiva}

Universidade Federal de São Carlos, São Carlos, São Paulo, Brasil.

\begin{abstract}
Resumo
Este estudo avaliou a influência de um programa de atividades físicas de longa duração nos sintomas depressivos de idosas depressivas e não depressivas. Participaram 40 mulheres, com idades entre 60 e 80 anos (média 66,8 e DP 7,03). Foi aplicada a Escala de Depressão para Idosos antes de iniciar a atividade física e após um ano. Os dados indicam que houve diminuição nos estados depressivos dos sujeitos com e sem depressão com idade entre 60 e 70 anos. Evidenciam também que o estado depressivo dos sujeitos com idade entre 70 e 80 anos permaneceu estável. Os resultados permitem concluir que a prática da atividade física influenciou positivamente no estado de depressão das idosas participantes.
\end{abstract}

Palavras-chave: Mulheres - Gerontologia - Atividade Física - Depressão.

\section{Introdução}

$\mathrm{E}^{\mathrm{s}}$ m 2025, 34 milhões de pessoas, ou seja, 15\% da população brasileira, estarão com idade superior a 60 anos e, nesse período, ocorrerá um aumento de 6,5\% de idosos ao ano e um decréscimo nos números absolutos de jovens entre 0 e 14 anos (GONÇALVES, 2001). Com o aumento crescente da população com idade superior a 60 anos, a geração de conhecimento sobre o processo de envelhecimento populacional e suas decorrências adquire significativa importância para o Brasil, a exemplo do ocorrido em países desenvolvidos.

O processo de envelhecimento, embora seja uma experiência universal e natural, tem sido relativamente estereotipado na trajetória histórica mundial. Desde o século XVI presencia-se o constante culto e adoração à juventude e à beleza, que resulta no preconceito contra a velhice (LORDA, 2001). Na sociedade brasileira, isso parece não ser diferente: o idoso usualmente enfrenta o preconceito, a desvalorização, o menosprezo e a exclusão social. A própria luta pelo retardamento ou anulação do envelhecimento influiu no contexto social e cultural que o idoso vivencia contemporaneamente. Okuma (1998) relata que em nossa sociedade o preconceito está presente de tal forma que ser velho e envelhecer recebem conotações negativas que se baseiam no declínio biológico natural com o envelhecimento.

Como resultado da realidade vivenciada pelo idoso, foi gerada uma minimização de seu papel social, o que teve como consequência transtornos psicológicos que, 
quando diagnosticados, demonstram alta prevalência de estados depressivos (GONZALEZ, 2001; SNOWDON, 2002). Estudos epidemiológicos (MCCRACKEN et al., 1997; WEYERER et al., 1995; VERAS, 1994; LIVINGSTON et al., 1990) mostram que a depressão é o distúrbio mental que apresenta maior frequência na terceira idade, além de ser considerado um dos distúrbios que mais geram gastos econômicos (CARVALHO; FERNANDES, 2002; UNUTZER et al., 1997). Outros trabalhos (HEPPLE; QUINTON, 1997; KENNEDY, 1996), também referem que $50 \%$ dos casos de suicídio na terceira idade são associados a diagnóstico de depressão.

Okuma (1998) relata que o termo depressão pode ter três diferentes contextos: ser relacionado a sintomas, a reações depressivas ou depressão reativa ou, ainda, à doença depressiva. No primeiro caso, trata-se de um estado de tristeza, ou humor alterado, com pequena duração e intensidade, sem interferir na vida de relação da pessoa, desaparecendo sem a necessidade de tratamento. As reações depressivas representam um conjunto de sinais e sintomas, como: tristeza, pessimismo, desânimo, transtornos de sono, de apetite e de sexualidade, e normalmente surgem após a ocorrência de uma patologia física ou psicológica significativa, ou de eventos existenciais, como fatos mortais, sociais, econômicos ou afetivos de difícil controle. Tais reações desaparecem após o fato estar controlado. Já a doença depressiva é uma condição de patologia com sinais e sintomas bem definidos pela hereditariedade, etiologia e pela patogenia da depressão, com consequências negativas para a vida de relação do indivíduo e apresenta respostas positivas a tratamentos medicamentosos.

No entanto, é preciso cuidado ao analisar e diagnosticar a doença depressiva em indivíduos com mais de 60 anos. A depressão apresenta-se na literatura com uma grande diversidade de significados, podendo ser encontrada com ênfase no estado emocional ou como uma patologia física. Muitas vezes o isolamento e a perda de autonomia que a pessoa deprimida se encontra são confundidos com as características da faixa etária (MAROTO, 2002; TRENTINI et al., 2005; SAMUELS et al., 2004; BALLONE; ORTOLANI; PEREIRA NETO, 2002). Fleck et al. (2003) relatam que a depressão é subdiagnosticada e subtratada, relatam que queixas somáticas são frequentes no próprio processo de envelhecimento normal, sendo que, nos idosos deprimidos, essas queixas são exacerbadas e associadas a alto índice de ansiedade. No entanto, os sintomas depressivos não são tão aparentes como na depressão em adultos (SAMUELS et al., 2004).

São muitos os estudos que direcionam sua atenção aos sintomas e doenças relacionados com a depressão, analisando a influência da atividade física nestes. Tais estudos apresentam a atividade física como preventivo e/ou minimizador dos sintomas depressivos e auxiliador no tratamento da doença depressiva. Dunn, Trivedi e O'neal (2001), Barbour e Blumenthal (2005) e Sjösten e Kivelä (2006) realizaram pesquisas bibliográficas e afirmam que a grande maioria dos estudos mostra que exercício físico tem influência positiva na depressão e/ou nos sintomas depressivos.

Farmer et al. (1988) avaliaram 1.900 indivíduos saudáveis com idade entre 25 e 77 anos e concluiram que a inatividade física pode ser um fator de risco para sintomas depressivos. 
Brown et al. (1995), para analisarem o efeito do exercício sobre a depressão, avaliaram 69 mulheres com idade média de 54,8 anos (DP 8,3 anos) e 66 homens com idade média de 50,6 anos (DP 8.0 anos). Os participantes foram divididos aleatoriamente em quatro grupos: grupo controle, grupo de caminhada de baixa intensidade, grupo de caminhada de baixa intensidade mais exercícios de relaxamento e grupo de Tai Chi Chuan. Todos os grupos foram avaliados previamente e após 16 semanas. As mulheres que participaram do grupo de Tai Chi Chuan obtiveram reduções significativas na tensão, no entanto não tiveram a detecção clínica da depressão por parte dos profissionais da saúde. Os homens com idade entre 65 e 74 anos e aqueles com mais de 85 anos apresentavam maior risco de estados depressivos.

Em uma pesquisa com 663 idosos, Lampinen et al. (2000) concluíram que quanto maior o tempo que o idoso não faz atividade física, maior será o risco de adquirir sintomas depressivos.

Babyak et al. (2000) estudaram 156 adultos e idosos que praticaram 10 meses de atividade física e concluíram que ela é uma eficiente estratégia para minimizar os sintomas depressivos, mesmo nas pessoas com doença depressiva já instalada.

Silverstein, Connor e Corbeau (2001) realizaram uma pesquisa transversal com 404 homens e 540 mulheres com idades entre 50 e 89 anos e relatam que o exercício foi significativamente associado com menores sintomas de depressão, no entanto concluem que o exercício não protege contra futuros sintomas depressivos.

Mather et al. (2002) também encontraram diferenças significativas nos sintomas depressivos entre idosos sedentários e idosos que participaram de 10 semanas de atividade física.

Strawbridge et al. (2002) avaliaram o efeito da atividade física na prevenção da depressão. Para isso, foi utilizada uma amostra de 1.947 indivíduos com idades de 50 a 94 anos, com acompanhamento de 5 anos e concluem que a hipótese é verdadeira.

Hollenberg, Haight e Tager (2003), em estudo com 1.246 mulheres idosas - sendo que 583 destas tem algum doença cardiovascular - relatam que as mulheres deprimidas mostraram níveis mais baixos em avaliação realizada por meio de caminhada em esteira. Os autores concluem que a depressão está associada à disfunção física e ao baixo desempenho em exercício de esteira das mulheres idosas.

Fukukawa et al. (2004) avaliaram os sintomas depressivos de 1.151 adultos e idosos no Japão e concluíram que os idosos pesquisados (idade entre 65 e 79 anos) que faziam atividade física regular apresentavam diminuição nos seus sintomas depressivos; no entanto, a mesma relação não foi encontrada em adultos de meia-idade (entre 40 e 64 anos).

O exercício físico e o relaxamento constituem formas de intervenção na depressão, na medida em que a atividade física reduz o risco da diminuição funcional e da mortalidade. Um programa de exercícios moderados melhora a saúde física e psicológica, pois aumenta a resistência, o número e a atividade do sistema imunológico, além de melhorar as condições comuns nos idosos, tais como osteoporose, doenças cardiovasculares e, ainda, o diabetes (GARCIA et al., 2006).

Nesse contexto, com o aumento crescente da população idosa e o alto índice de estados depressivos nesta população, há a necessidade de entender melhor os vínculos 
entre a atividade física e a presença de sintomas depressivos. Ainda faltam estudos que avaliem a diferença da influência da atividade física sobre os estados depressivos de indivíduos idosos com e sem diagnóstico clínico de depressão. Este estudo busca minimizar esta lacuna e teve como objetivo verificar a influência de um programa de atividades físicas de longa duração sobre sintomas de depressão em mulheres com 60 anos ou mais.

\section{Método}

\section{Participantes}

Participaram 40 mulheres com idade entre 60 e 80 anos (média=66,8 e DP 7,03), residentes no município de São Carlos. O programa de atividades físicas foi divulgado por meio de jornais e televisão da região. Primeiramente, as participantes constituíram dois grupos identificados por meio de uma entrevista inicial: Grupo D (constituído por 11 idosas com diagnóstico médico e que utilizavam medicamento antidepressivo), Grupo ND constituído por 29 mulheres sem história de aspectos depressivos e que não tomavam medicamento antidepressivo. Todas idosas com características compatíveis para formar o Grupo D faziam parte da faixa etária de 60 a 69 anos (média 63,8 e +-3,37). Como o Grupo ND apresentou um número superior ao Grupo D, para melhor análise dos resultados, o Grupo ND foi subdividido em dois novos grupos: Grupo ND+ (grupo de 10 idosas com idade de 70 a 79 anos, média 73 e DP 5,16) e o Grupo ND- (constituído de 11 idosas com idade entre 60 e 69 anos, média 63,18 e +-2,08). Os critérios de inclusão levaram em conta: exame médico recente, não existência de patologias músculo-esqueléticas ou orgânicas que impedissem a execução de atividades físicas e disponibilidade pessoal. Foram excluídas as participantes que não fizeram as duas avaliações ou que não estiveram presentes em pelo menos $75 \%$ das aulas. Todas as participantes assinaram o Termo de Consentimento Livre e Esclarecido, e o estudo foi aprovado pelo Comitê de Ética da UFSCar, sob no 190/2006.

\section{Medidas e procedimentos}

Para avaliar os sintomas da depressão, foi utilizada a Escala de Depressão para Idosos de Fiatarone e Nelson (1996) que é validada para a população brasileira. Este teste consta de 30 questões relacionadas com a satisfação de vida e estados de ânimo do indivíduo (MATSUDO, 2000). A correção do teste leva em conta o número total de pontos negativos, ou seja, todas aquelas respostas que estejam associadas a sentimentos de depressão. Quanto maior o número de pontos negativos, maior será o estado depressivo. Este questionário permite avaliar situações que demonstram sintomas da depressão (OKUMA, 1998), inclusive em pessoas sem o quadro da depressão já instalado.

A entrevista inicial foi realizada por um profissional da saúde que detectou os indivíduos que utilizavam medicamento antidepressivo e os não portadores de histórico depressivo. Em seguida, antes do início do programa de atividades físicas, ambos 
os grupos foram submetidos à primeira aplicação do questionário (M1). Após um ano de aplicação do programa, foi novamente realizada a medida de aspectos depressivos por meio do mesmo questionário (M2).

\section{A intervenção}

O programa de atividades físicas teve início em abril de 2005 e foi realizado em 123 sessões divididas em: 10 semanas de atividade, seguidas por três semanas de descanso (que coincidiram com as férias de julho de 2005), depois mais 21 semanas de atividade, quatro semanas de descanso e, por fim, 11 semanas de atividade. Cada sessão teve a duração de 50 a 55 minutos e foi realizada três vezes por semana nas segundas, quartas e sextas-feiras. A sessão consistiu de um pré-aquecimento por meio de exercícios de leve intensidade de aproximadamente três minutos; mais uma parte composta de exercícios de alongamentos miofasciais passivos dos principais grupos musculares (posteriores da perna e coxa, anteriores da coxa e pelve, vertebrais, paravertebrais, peitorais, etc.) de aproximadamente cinco minutos; de ativação cardiocirculatória aeróbia por meio de caminhadas ou exercícios combinados ou ainda de uma corrida leve com duração de 12 minutos; exercícios adaptados de força, potência e resistência com alteres de mão de um e dois quilos com duração de 10 a 15 minutos; atividades de coordenação, agilidade e flexibilidade (12 min), exercícios respiratórios e de relaxamento (cinco a sete min). As sessões foram ministradas por educadores físicos previamente treinados e auxiliados por assistentes.

\section{Análise dos Dados}

Para a análise estatística dos dados gerais dos escores do Grupo D e do Grupo ND, foi utilizado o Teste T-Pareado. Este mesmo teste foi utilizado para análise dos escores entre M1 e M2 dos grupos. Para os cálculos das diferenças entre os subgrupos divididos em faixas etárias (Grupo ND+ e Grupo ND-), foi utilizado o teste de Wilcoxon. O nível de significância adotado, ambos os casos, foi p 0,05.

\section{Resultados}

Os resultados obtidos em M1 no Grupo D (12.90909) e em M1 no Grupo ND (7.5862) mostraram diferença estatisticamente significativa entre os escores ( $p$ $=0.0409)$. No entanto, os resultados obtidos entre M2 do Grupo D (8.090909) e M2 do Grupo ND (5.7586) apresentam igualdade entre os grupos ( $p=0.1681)$. Estes dados sugerem que os grupos D e ND eram diferentes na primeira avaliação e se tornaram iguais após um ano de intervenção de atividade física.

A Tabela 1 mostra a diferença entre as médias dos escores obtidos em M1 e M2 nos grupos D, ND- e ND+. Os dados indicam que houve diferença significativa entre as médias da primeira e da segunda avaliações tanto no grupo D quanto no grupo ND-. Mostrou também que não houve diferença estatisticamente aceitável entre M1 e M2 no grupo ND+. 
Tabela 1 - Médias amostrais dos Grupos D, ND- e ND+ e o nível de significância entre M1 e M2 nos três grupos.

\begin{tabular}{|l|l|l|l|l|}
\hline Grupos & N & Medida 1 & Medida 2 & $(\mathbf{p})$ bilateral \\
\hline D & 11 & 12.90909 & 8.090909 & $0.0294^{*}$ \\
\hline ND- & 19 & 6.947368 & 4.947368 & $0.0132^{*}$ \\
\hline ND+ & 10 & 8.8 & 7.3 & 0.6726 \\
\hline
\end{tabular}

*Significância para $\mathrm{p}=0,05$

\section{Discussão}

Inicialmente é importante destacar que aproximadamente 30\% dos participantes apresentaram quadro depressivo e fazem uso de medicamentos. Tal aspecto vai ao encontro dos resultados de outros trabalhos (GONZALEZ, 2001; SNOWDON, 2002; MCCRACKEN et al., 1997; WEYERER et al, 1995; VERAS, 1994; LIVINGSTON et al., 1990), que evidenciam a alta frequência da depressão em idosos.

Outro aspecto a ser ressaltado é o fato da atividade física ter sido mais eficaz para as mulheres de 60 a 69 anos que para as de 70 a 79 (embora estas últimas também apresentem tendência à diminuição das características depressivas após a intervenção).

Os resultados também indicam, de uma forma geral, que a prática da atividade física tem influência nos sintomas depressivos de idosas, independente da presença de um quadro depressivo diagnosticado. Isto pode ser notado pelo fato dos grupos $\mathrm{N}$ e ND- apresentarem diferenças estatisticamente significativas entre as medidas M1 e M2. Tais dados são concordantes com as observações de Okuma (1998) que realizou uma revisão abordando 12 estudos que analisavam a depressão e a atividade física em pessoas de 17 a 60 anos, e relata que todos os estudos concluem que a atividade física apresenta maior efeito antidepressivo que qualquer outro tratamento, tendo o mesmo valor que o tratamento psicoterápico. Outros trabalhos também relatam que a atividade física é um fator pertinente no tratamento do estado depressivo (OURIQUES; FERNANDES, 1997; GOBBI, 1997). Andrade (2000) realizou um estudo com 16 mulheres com mais de 60 anos que fizeram atividade aquática por seis meses e verificou a diminuição do estado depressivo em 15 indivíduos. Lindwall et al. (2007) avaliaram o estado depressivo de 860 homens e mulheres de 60 a 96 anos e concluíram que os idosos inativos apresentam nível de depressão superior aos que praticam atividade física. Penninx et al. (2002) avaliaram a relação entre exercícios e sintomas depressivos comparando exercícios resistidos e exercícios aeróbios e chegaram à conclusão de que ambos diminuem o estado de depressão.

No que tange à associação de atividades físicas com medicação antidepressiva, parece ainda haver certa lacuna na literatura. Okuma (1998) já relatava que o número de estudos ainda era relativamente pequeno e que existiam muitas questões a serem abordadas, além de faltar estudos que relacionem a atividade física e o uso de medicamentos no tratamento da depressão.

As limitações do presente estudo estão no fato de não terem sido discriminados os tipos de medicação utilizada, no pequeno número de sujeitos em cada grupo e na ausência de um grupo que não realizasse atividade física, para melhor contraste dos 
resultados. Nessa direção, sugere-se a realização de novos trabalhos com o controle dessas variáveis e com delineamento mais completo.

\title{
Conclusão
}

Conclui-se que o programa de atividades físicas de longa duração influenciou positivamente nos estados depressivos das mulheres idosas participantes, sendo que as idosas que não tomam medicamento antidepressivo apresentaram, no início do programa de atividade física, um escore maior que as idosas que não apresentam diagnóstico de doença depressiva. Após um ano de atividade física, os dois grupos tornaram-se estatisticamente iguais. O programa de atividade física influenciou diminuindo os estados depressivos em todas as idosas com idade entre 60 e 69 anos, e estabilizando esse estado nas idosas com 70 ou mais anos.

Influence of a Long Term program of Physical Activities on Symptoms of Depression in the Aged

\begin{abstract}
This study evaluated the influence of a long term program of physical activities on symptoms of depression in depressed and non-depressed elderly individuals. Forty women, aged between 60 and 80 (mean of 66.8 and SD of 7.03) took part in the study. The Depression Scale for the Elderly was applied before starting the physical activities and one year later. The data indicated there was a decrease in the depressive state of subjects with and without depression and aged between 60 and 70 . It was also shown that the depressive state of subjects between 70 and 80 years of age remained stable. The results allowed for the conclusion that the practice of physical activity positively influenced the depressive state of the elderly participants.
\end{abstract}

Keywords: Women - Gerontology - Physical Activity - Depression.

Influencia de un Programa de Actividades Físicas de larga duración sobre las señales depresivas en adultas mayores

\section{Resumen}

Este estudio evaluó la influencia de un programa de actividades físicas de Larga Duración en los síntomas depresivos de mujeres adultas mayores depresivas y no depresivas. Participaron 40 mujeres, con edades entre 60 y 80 años (promedio de 66,8 y DP 7,03). Fue aplicada la Escala de Depresión para Ancianos antes de iniciar la actividad física y después de un año. Los datos indican que hubo disminución en los estados depresivos de los sujetos con y sin depresión con edad entre 60 y 70 años. Se evidencia también que el estado depresivo de los sujetos con edad entre 70 y 80 años permaneció estables. Los resultados permiten concluir que la práctica de la actividad física influenció positivamente en el estado de depresión de las mujeres adultas mayores participantes.

Palabras clave: Mujeres - Gerontología - Actividad Física - Depresión.

\section{Referências}

ANDRADE, A.D. Benefícios da atividade física no meio aquático para idosos depressivos. Revista A Terceira Idade. SESC: São Paulo, 14 (27), 2003. 
BABYAK, M.; et al. Exercise treatment for major depression: maintenance of therapeutic benefit at 10 months. Psychosomatic Medicine n. 62, p. 633-638 American Psychosomatic Society 2000

BALLONE, G. J.; ORTOLANI, I.V.; PEREIRA NETO, E. Da emoção á lesão. São Paulo: Manole, 2002.

BARBOUR, K. A.; BLUMENTHAL, J.A. Exercise training and depression in older adults. Psychosomatic Medicinev. 26, issue 1, supplement 1, p. 119-123, dec. 2005

BROWN, D. R.et al. Chronic psychological effects of exercise and exercise plus cognitive strategies. Med Sci Sports Exerc. n.27, v.5, p. 765-75, may. 1995

CARVALHO, V. F. C.; FERNANDEZ, M. E. D. Depressão no idoso. In: NETTO, M. P. Gerontologia: a velhice e o envelhecimento em visão globalizada. São Paulo: Atheneu; 2002. p. 160-173.

DUNN, A. L.; TRIVEDI, M. H.; O'NEAL, H. A.; Physical activity dose-response effects on outcomes of depression and anxiety. Med Sci Sports Exerc. n. 33 (6 Suppl), p. 587-97; discussion 609-10, jun. 2001

FARMER, M. E.et al. Physical activity and depressive symptoms: the nhanes i epidemiologic follow-up study. American Journal of Epidemiology. v. 128, n. 6, p. 1340-1351, 1988.

FIATORE, M. A.; NELSON, M. E. Depresión scale for the elderly. Boston. (Baseline assessment package of the United States Department of Agriculture Human Nutrition Research Center on Aging at Tufts University), 1996.

FLECK, M. et al. Diretrizes da associação médica brasileira para o tratamento da depressão (versão integral). Rev. Brasileira de Psiquiatria, vol.25 no.2, p. 114-122, jun. 2003.

FUKUKAWA, Y.et al. Age differences in the effect of physical activity on depressive symptoms. Psychol Aging. n.19, v.2: p. 346-51, jun. 2004

GARCIA, A. et al. A depressão e o processo de envelhecimento. Ciências \& Cognição. ano 03, v. 07, p.111-121, 2006.

GOBBI, S. Atividade física para pessoas idosas e recomendação da Organização Mundial de Saúde 1996. Revista Brasileira de Atividade Física e Saúde, v. 2, n. 2, p. 41-49, 1997.

GONÇALVES, A. K. Novo ritmo da terceira idade. In: Pesquisa Fapesp, n. 67, p. 68, Ag. 2001.

GONZÁLEZ, C. M. Depresión en ancianos: un problema de todos. Rev. Cuba. Med. Gen. Integr. n.17, v.4, p. 316-320, jul.-ago. 2001.

HEPPLE J.; QUINTON C. One hundred cases of attempted suicide in the elderly. Br J Psychiatry 1997. n.171: p. 42-46, 1997. 
HOLLENBERG, M.; HAIGHT, T.; TAGER, I. B.; Depression decreases cardiorespiratory fitness in older women. J Clin Epidemiol. n.56, v.11, p. 1111-7, nov. 2003

KENNEDY, G. J. Suicide and depression in late life: critical issues in treatment, research, and public policy. New York: John Wiley; 1996.

LAMPINEN, P.; HEIKKINEN, R. L.; RUOPPILA, I. Changes in intensity of physical exercise as predictors of depressive symptoms among older adults: an eightyear follow-up. Preventive Medicine v. 30, Issue 5, p. 371-380, may, 2000.

LINDWALL, M. et al. Depression and exercise in ederly men and women: findings from the swedish national study on aging and care. J Aging Phys Act, n.1, v.1, p. 41-55, 2007.

LIVINGSTON, G. et al. The gospel oak study: prevalence rates of dementia, depression and activity limitation among elderly residentes in inner London. Psychol Med n.20, p. 137-146, 1990.

LORDA, C. R. Recreação na terceira idade. 3. ed. Rio de Janeiro:: Sprint, 2001.

MAROTO, G. N. V. Depressão e sociedade: estudo das representações sociais da depressão em um grupo de pessoas adultas da cidade de São Carlos. 268p., Tese (Doutorado em Ciências Médicas). Unicamp, Campinas: SP: 2 vol., 2002.

MATHER, A. S.; et al. Effects of exercise on depressive symptoms in older adults with poorly responsive depressive disorder Randomised controlled trial, The British Journal of Psychiatry. N.180, p. 411-415, 2002

MATSUDO, S. M. M. Avaliação do idoso: física e funcional. Londrina: Midiograf, 2000 .

MCCRACKEN, C. F. M. et al. Prevalence of dementia and depression among elderly people in black and ethnic minorities. Br J Psychiatry. 171, p. 269-73, 1997.

OKUMA, S. S. O idoso e a atividade física: fundamentos e pesquisa. Campinas, SP: Papirus, 1998.

OURIQUES, E. P. M.; FERNANDES, J. A. Atividade física na terceira idade: uma forma de prevenir a osteoporose? Revista Brasileira de Atividade Física e Saúde, v. 2, n. 1, 53-59, 1997.

PENNINX, B. W. J. H. et al. Exercise and depressive symptoms: a comparison of aerobic and resistance exercise effects on emotional and physical function in older persons with high and low depressive symptomatology. The Journals of Gerontology, $n .57$, p.124-132, 2002.

SAMUELS, S. et al. Depression in autopsy-confirmed dementia with lewy bodies and alzheimer's disease. Mount Sinai. J. Med., n.71,v.1, p. 55-62, 2004.

SILVERSTEIN, D. K.; CONNOR, E. B.; CORBEAU, C. Cross-sectional and prospective study of exercise and depressed mood in the elderly. American Journal of Epidemiology, v. 153, n. 6, p. 596-603, 2001. 
SJÖSTEN, N.; KIVELÄ, S.L. The effects of physical exercise on depressive symptoms among the aged: a systematic review. International Journal of Geriatric Psychiatry v. 21, Issue 5 , p. 410-418, may, 2006.

SNOWDON, J. Qual é a prevalência de depressão na terceira idade?. Rev. Bras. Psiquiatr., v.24 supl.1, p .42-47, 2002.

STRAWBRIDGE, W. J.et al. Physical activity reduces the risk of subsequent depression for older adults. American Journal of Epidemiology. v. 156, n. 4, 2002

TRENTINI, C. et al The influence of somatic symptons on the performance of elders in the Beck Depression Inventory. vol.27, n. 2, São Paulo, Junho, 2005.

UNUTZER, J. et al. Depressive symptoms and the cost of health services in HMO patients aged 65 years and older: a 4-year prospective study. J Amer Med Assoc, n.277, p. 1618-1623, 1997.

VERAS, R. P. País jovem com cabelos brancos. Rio de Janeiro: Relume Dumará, 1994.

WEYERER, S. et al Prevalence and course of depression among elderly residential home admissions in Mannheim and Camden, London. Int Psychoger, n.7, p. 47994, 1995.

Recebido em: 14/09/2009

Revisado em: 05/10/2009

Aprovado em: 04/11/2009

Endereço para correspondência

Vanessa Helena Santana Dalla Déa

Universidade Federal de Goiás

Faculdade de Educação Física

Campus II, Caixa Postal 131

CEP 74001-970, Goiânia-GO, Brasil

E-mail:vanessasantana@ig.com.br 